Cahiers de recherches médiévales

\title{
Le Charlemagne de Girart d'Amiens et la tradition épique française
}

Alain Corbellari

\section{(2) OpenEdition \\ 1 Journals}

Édition électronique

URL : https://journals.openedition.org/crm/2678

DOI : $10.4000 / \mathrm{crm} .2678$

ISSN : 1955-2424

Éditeur

Honoré Champion

\section{Édition imprimée}

Date de publication : 15 décembre 2007

Pagination : 189-199

ISSN : 1272-9752

\section{Référence électronique}

Alain Corbellari, «Le Charlemagne de Girart d'Amiens et la tradition épique française », Cahiers de recherches médiévales [En ligne], 14 | 2007, mis en ligne le 15 décembre 2010, consulté le 15 décembre 2022. URL : http://journals.openedition.org/crm/2678 ; DOI : https://doi.org/10.4000/crm.2678 


\section{RM}

\section{Le Charlemagne de Girart d'Amiens et la tradition épique française}

La publication du Charlemagne de Girart d'Amiens était depuis plus d'un siècle l'un des fantasmes les mieux établis de la critique médiévale: comme si la prophétie de Gaston Paris, selon qui il était «inédit et ne sera jamais publié » ${ }^{1}$ avait eu valeur d'interdit pour les philologues trop téméraires, la longueur de l'attente de son édition critique n'aura eu d'égale que l'ampleur de sa réputation d'ennui : «le goût de l'époque était mauvais et l'écrivain pire encore», nous avait charitablement prévenu l'auteur de l'Histoire poétique de Charlemagne. Mais les suspenses les plus insoutenables finissent toujours par trouver leur résolution et voici que l'édition de Daniel Métraux ${ }^{2}$ vient mettre fin à un mythe qui n'avait que trop duré. Pouvons-nous toujours, maintenant que nous avons la possibilité d'en juger sur pièce, soutenir la thèse de l'effroyable médiocrité littéraire d'un auteur qui pourrait bien être sinon le plus prolifique (dans le sens où l'on distinguerait par ce terme le nombre d'œuvres différentes nées de sa plume), du moins le plus abondant de la littérature en ancien français? Rappelons que le Charlemagne (23.348 vers), commande de Charles de Valois, frère de Philippe le Bel, couronne sans doute une carrière brillante d'écrivain appréciablement stipendié, ce dont on peut juger au fait que le roman arthurien d'Escanor (25.938 vers), écrit vers $1280^{3}$, est dédié à Aliénor de Castille, descendante de la comtesse de Champagne qui, un siècle auparavant, avait accueilli Chrétien de Troyes, et que le roman merveilleux de Meliacin (19.159 vers) ${ }^{4}$ semble dédié à Gaucher de Châtillon, sénéchal de Philippe le Bel, et à son épouse Isabelle de Dreux.

Il paraît difficile de déceler, dans les trois œuvres-fleuves de Girart, les traces d'une «personnalité » littéraire au sens moderne du terme ${ }^{5}$, revendication dont il est

\footnotetext{
${ }^{1}$ Gaston Paris, Histoire poétique de Charlemagne, Paris, Franck, 1865, p. 95.

${ }^{2}$ Girart d'Amiens, L'Ystoire le Roy Charlemagne, éd. par Daniel Métraux, Lewiston / New York, The Edwin Mellen Press, 2004 (3 vol.).

${ }^{3}$ Girart d'Amiens, Escanor, roman arthurien en vers de la fin du XIII ${ }^{e}$ siècle, éd. Richard Trachsler, Genève, Droz, «Textes littéraires français », 2 vol., 1994.

${ }^{4}$ Girart d'Amiens, Meliacin ou le Cheval de Fust, éd. Antoinette Saly, Aix-en-Provence, CUERMA (Senefiance $\left.\mathrm{n}^{\circ} 27\right), 1990$.

5 Ainsi l'étude du vocabulaire ne donne-t-elle que des résultats assez décevants. De la vingtaine d'hapax repérés par Antoinette Saly dans le Meliacin (Antoinette Saly, "Le Vocabulaire de Girart d'Amiens dans le Meliacin ", Actes du XVIII Congrès International de Linguistique et de Philologie romane, Trèves, 1986, Tübingen, Niemeyer, 1989, t. IV, p. 401408), on n'en retrouve qu'un dans le Charlemagne: ostruance, qui est toutefois un mot fort intéressant, car il pourrait être le signe d'un intérêt de Girart pour l'astrologie. Ce mot, en effet, qui semble signifier «destin déterminé par les astres, chance, heureuse fortune» (A. Saly, art. cit.) et comprend la même racine astr- (devenue ostr-) que malotru (malostru), témoigne incontestablement d'une vision très personnelle du destin des héros que Girart met en scène (le mot est toutefois absent de l'Escanor). Faut-il relier au même intérêt pour les
}

Cahiers de Recherches Médiévales, 14, 2007 
de surcroît malaisé de dire si elle est ou non anachronique, dans la mesure où Albert Henry a cru pouvoir discerner un style propre à Adenet le Roi ${ }^{6}$, peut-être maître et à coup sûr concurrent de Girart, puisque son Cléomadès, inspiré comme Méliacin du conte oriental du cheval d'ébène, lui a été commandé à la même époque par la même mécène et que les deux auteurs semblent bien avoir été en l'occurrence dans la même position que Racine et Corneille face au sujet de Titus et Bérénice? . L'aspect défavorable de la comparaison serait un nouvel argument pour postuler le moindre talent de Girart, mais tel n'est pas ici notre but. Plus intéressant, en effet, nous semble, avant tout jugement définitif sur Girart écrivain, de s'interroger sur l'usage qu'il fait de ses sources : de ce point de vue, Girart apporte un sang très réellement neuf à la vieille épopée française.

On pouvait estimer le débat clos depuis les travaux déjà anciens de Gérard Brault ${ }^{8}$, mais si ce dernier met exemplairement en valeur le rôle des chroniques (en particulier des Grandes Chroniques de France dans la traduction de Primat de 1274) dans l'élaboration du Charlemagne, il est beaucoup plus succinct quant aux sources proprement épiques, donc traditionnelles et littéraires, de Girart. Or, la comparaison des chansons de la "geste du roi» avec le texte du Charlemagne offre bien des surprises, dont on tentera ici de donner un petit aperçu.

Une première remarque s'impose, presque trop évidente : le Charlemagne est une chanson de geste biographique, la seule, du moins dans la littérature française' ${ }^{9}$ à prendre en compte toute la vie du grand empereur, ce qui contredit l'un des principes fondamentaux des chansons de geste françaises, puisque Charles, comme

astres le très long développement sur les signes météorologiques que Girart glisse dans le livre II du Charlemagne (v. 12.472-12529) et l'amplification du motif du deuil de la nature au moment de la mort de Roland (livre III, v. 23.075-23.089)? Il convient sans doute de rester prudent sur ce point.

${ }^{6}$ Comme on le sait, Albert Henry commence sa grande éditions des Euvres d'Adenet le Roi (Bruges, De Tempel, 1951, t. I, p. 11-64), par une monumentale biographie de cet auteur.

${ }^{7}$ Voir Antoinette Saly, «Les Destinataires du roman de Meliacin», Travaux de Linguistique et de Littérature, XIX, 2 (1981), p. 7-16 et «Écrivains mystificateurs : le cas d'Adenet le Roi et de Girart d'Amiens », repris dans Images, structures et sens : études arthuriennes, Aix-enProvence, CUERMA, 1994, p. 245-253.

${ }^{8}$ Gerard J. Brault, "The Alleged Sources of Girart d'Amiens's Charlemagne ", Modern Language Notes, 74 (1959), p. 412-416 et «Girart d'Amiens and the Pseudo-Turpin Chronicle », Zeitschrift für romanische Philologie, 76 (1960), p. 64-93.

${ }^{9}$ La seule version poétique de la vie du grand empereur en ancien français avant Girart, est la Chronique rimée de Philippe Mousket, que l'on ne peut pas à proprement parler appeler une chanson de geste. On sait par ailleurs que la Karlamagnus-Saga scandinave propose également un parcours complet de la vie du grand empereur, mais plutôt qu'un récit unifié, il s'agit là d'une collection de récits, inspirée des chansons de gestes françaises (dont les originaux ne nous sont d'ailleurs pas tous parvenus) et organisée de manière assez lâche (voir Paul Aebischer, Textes norrois et littérature française du Moyen Âge, Genève, Droz, 19541972, 2 vol. et Daniel W. Lacroix, La Saga de Charlemagne: traduction française des dix branches de la "Karlamagnus saga» norroise, Paris, Le livre de poche, «Classiques modernes », 2000). 
$\operatorname{Arthur}^{10}$, apparaît plus comme le garant, par son identification à l'univers qu'il régit, que comme le protagoniste même de l'aventure; il est celui à la cour de qui les choses peuvent advenir et n'occupe, par là, que très occasionnellement le devant de l'action. Seule fait exception la chanson de Mainet ${ }^{11}$ qui nous présente un Charles encore bachelier (un juvenis, aurait dit Duby), donc non revêtu des insignes de pouvoir qui le fige, dans les autres chansons, dans sa posture impériale ${ }^{12}$. Dans la tradition arthurienne, seul le roman fondateur, le Brut, qui se voulait d'abord une chronique rimée, narrait dans son entier la vie du grand roi breton, toutes les végétations romanesques qui se sont greffées sur ce premier tronc ayant bien pris soin de diluer la vie du souverain dans le récit, infiniment plus complexe, de celles de ses compagnons. Cette prégnance du dispositif de la chronique dans le récit biographique royal se retrouve dans l'œuvre de Girart d'Amiens, à la différence que si, dans la tradition bretonne, le roman s'épanouissait en débordant la chronique de toutes parts, la matière de France effectue, de son côté, une manière de retour aux sources en «retrouvant», peu avant son exténuation, le récit chronologique que la littérature vernaculaire n'avait jamais produit.

Girart d'Amiens divise la vie de l'empereur à la barbe fleurie en trois phases, chacune décrite dans l'un des trois livres, de longueur sensiblement égale, du Charlemagne.

Le PREMIER LIVRE (7690 vers) raconte les enfances de Charles. La matière reprend, selon toute probabilité, de près, en l'amplifiant, la chanson de Mainet, dont, rappelons-le, ne nous restent que 900 vers. Le rôle des chroniques est ici réduit, pour ne pas dire nul, car on sait que cet épisode doit fort peu à la jeunesse réelle de Charlemagne : brodant sur le thème populaire de l'éviction de l'héritier légitime par des traîtres qui finiront évidemment par être démasqués et punis ${ }^{13}$, il s'inscrit parfaitement dans le cadre des épreuves qualifiantes liées au mythe universel de la naissance du héros, dont les composantes ont jadis été dégagées par Rank ${ }^{14}$, et sur les origines exactes duquel on s'interroge toujours.

Le DEUXIEME LIVRE (8586 vers) est le moins unifié; il semble d'ailleurs inachevé et pose la question du degré de complétude du Charlemagne: non seulement ce deuxième livre se termine en queue de poisson, mais il dénote un net

\footnotetext{
${ }^{10}$ Voir Dominique Boutet, Charlemagne et Arthur ou le roi imaginaire, Paris, Champion, 1992.

${ }^{11}$ Mainet, fragment édité par Gaston Paris, Romania, 4 (1875), p. 303-337 (à compléter par Charles Samaran, « Lecture sous les ultra-violets», Romania, 53 (1927), p. 291-297).

${ }^{12}$ On sait que la secondarité fonctionnelle, au niveau de l'action, du personnage du souverain fera évoluer ce dernier vers des rôles de plus en plus passifs (que l'on songe à l'Arthur faible et indécis du Perceval de Chrétien de Troyes) ou carrément négatifs: c'est éminemment le cas du Charlemagne irascible et violent mis en scène par les chansons du cycle « de la révolte ».

13 Joseph Bédier («La Légende des 'Enfances' de Charlemagne et l'histoire de Charles Martel », Les Légendes épiques, Paris, Champion, t. III, 1917, p. 1-38) y avait bien reconnu la transposition légendaire de l'enfance non de Charlemagne mais de son grand père homonyme (avec qui certaines chansons de geste le confondent d'ailleurs), Charles Martel.

${ }^{14}$ Otto Rank, Le Mythe de la naissance du héros, trad. française, Paris, Payot, 1983 (original allemand 1909).
} 
relâchement des règles métriques que s'était données Girart ${ }^{15}$. En fait, tout se passe comme si ce livre avait été composé en dernier et laissé inachevé suite à un événement qui est peut-être tout simplement la mort de son auteur. C'est le plus historique des trois livres; il évoque les guerres de Saxe, les campagnes de Lombardie et d'Espagne et se termine sur le projet de croisade en Terre sainte. La source essentielle en est Primat.

Le TROISIEME LIVRE (7072 vers), enfin, est consacré à la croisade espagnole et culmine dans le récit de la bataille de Roncevaux. Texte phare et fondateur de toute la matière de France, La Chanson de Roland est utilisée, par un juste retour des choses, pour couronner l'œuvre qui clôt cette tradition: chez Girart, la mort de Roland ne précède que de peu celle du vieil empereur qui, après avoir glorieusement vengé son neveu, revient mourir, ou plutôt disparaître emporté par des anges, à Aixla-Chapelle, dans une atmosphère d'Apocalypse empruntée de toute évidence à la fameuse scène des signes prémonitoires de La Chanson de Roland ${ }^{16}$. Selon Gerard Brault tout ce livre n'est «qu'une version rimée de la Chronique du pseudoTurpin ${ }^{{ }^{17}}$; mais le problème ne saurait être résolu aussi facilement. La brièveté du célèbre apocryphe contraste avec les 8000 vers de la troisième partie du Charlemagne, et il est très difficile d'imaginer que Girart ne se soit pas du tout inspiré des nombreuses traditions épiques qui circulaient de son temps. Au début du $\mathrm{XIV}^{\mathrm{e}}$ siècle, toutes les chansons que nous connaissons sur l'expédition espagnole de Charlemagne n'ont pas encore été écrites (La Prise de Pampelune, par exemple, n'est sans doute pas antérieure à 1350) et c'est donc sur une tradition vivace que s'ente le poème de Girart.

Cependant, la comparaison avec des poèmes et des fragments existants s'avère décevante : il paraît, par exemple, impossible, de déduire la moindre parenté entre le Charlemagne et le seul fragment retrouvé de la chanson d'Agolant ${ }^{18}$. L'épisode conservé de ce dernier texte fait état d'une lutte furieuse entre Ogier et le roi Sarrasin Agolant, qui est une première fois désarçonné, avant de repartir à la charge; cette scène n'a pas de correspondant dans la Chronique de Turpin et Paul Meyer en tirait argument pour faire descendre les deux textes d'une source commune perdue. Hélas, l'épisode ne se trouve pas non plus dans le Charlemagne. Par ailleurs, l'Agolant fait intervenir, dans l'armée de l'empereur, deux personnages que la Chronique de Turpin ne mentionne pas et qui ne sont autres que des

\footnotetext{
${ }^{15}$ Par exemple, selon Daniel Métraux, dont on consultera l'édition pour plus de détails, seules $79 \%$ des laisses du livre II respectent l'alternance des rimes masculines et féminines contre respectivement $100 \%$ et $94 \%$ dans les livres I et III. Signalons que le fait avait déjà été remarqué par Gaston Paris (Histoire littéraire de la France, 31, 1893, p. 204), qui n'en maintenait pas moins l'idée que la fin du deuxième livre était sans doute simplement perdue.

${ }^{16}$ Celle-ci faisant elle-même écho au récit biblique de la mort du Christ, on assiste ici à un enchaînement intertextuel typique de l'écriture du Moyen Âge tardif: on n'écrit plus seulement sur le déjà écrit, mais on se plaît à souligner l'épaisseur d'une tradition en renchérissant sur les topoï les mieux établis.

${ }^{17}$ G. J. Brault, "Girart d'Amiens », notice du Dictionnaire des Lettres françaises, Paris, Fayard, «Pochothèque », 1992, p. 545-546.

${ }^{18}$ Paul Meyer, «Fragment d'une chanson de geste relative à la Guerre d'Espagne », Romania, 35 (1906), p. 22-31.
} 
transfuges de la geste de Guillaume, puisqu'il s'agit de Bernard de Brabant et de Guerin d'Anseüne. Nouveau manque de chance : le Charlemagne ne connaît pas non plus ces personnages.

Cette piste de recherche s'avérant donc, jusqu'à nouvel avis, une impasse, c'est aux rapports conflictuels des matières épique et historique que nous consacrerons l'essentiel des remarques qui vont suivre; nous allons voir que l'examen du texte de Girart n'est peut-être pas sans offrir des perspectives intéressantes sur la vision du monde d'un écrivain de l'époque de Philippe Le Bel.

L'utilisation des sources historiques fait que, malgré sa forme en alexandrins et laisses rimées inégales ${ }^{19}$, le Charlemagne tient tout autant, sinon par places davantage, de la chronique rimée que de la chanson de geste : la rapidité de l'action, dans le deuxième livre en particulier, nous éloigne beaucoup du chronotope habituel de la chanson de geste. À la guerre «de positions» qu'évoquent généralement les textes épiques, dont l'action se réduit volontiers à des combats rapprochés autour d'un même site ${ }^{20}$, s'oppose ici une suite vertigineuse de campagnes, de Blitzkriege serions-nous tentés de dire, parfois décrites en quelques lignes ${ }^{21}$. De manière générale, Girart montre un souci de la cohérence chronologique qui, même encore balbutiant, tranche avec l'optique de ses devanciers. Bien sûr, légende et histoire ne sont toujours pas clairement distinguées pour lui et, comme pour tous les hommes de son temps, la chanson de geste est encore l'histoire même; elle se trouve cependant concurrencée par la chronique et on sent bien, sans que l'une soit systématiquement privilégiée par rapport à l'autre, que commence à poindre ici l'idée de la nécessité $\mathrm{du}$ choix entre versions contradictoires. À l'instar de l'idée de ressemblance physique dans le portrait (qui ne commence que de timidement émerger précisément à la même époque avec Giotto ${ }^{22}$, la vraisemblance en matière d'histoire, sans être un concept totalement inconnu, reste une notion dont on aperçoit encore mal l'utilité au regard de l'efficacité pragmatique d'une narration exemplaire.

Mais prenons quelques exemples : si nous voulons voir Girart aux prises avec les problèmes que lui posent ses sources, c'est surtout dans le deuxième livre que se feront le mieux jour les contradictions entre les biographies réelle et légendaire du premier empereur d'Occident. Rimant l'histoire de l'expédition espagnole de 778, Girart narre sans sourcilier le récit suivant :

\footnotetext{
${ }^{19}$ Inégalité contrebalancée, dans le premier livre, par un visible effort pour faire alterner, à la manière d'Adenet le Roi, laisses féminines et masculines d'une vingtaine de vers.

${ }^{20}$ Voir La Chanson des Saisnes de Jean Bodel qui se déroule de part et d'autre de la Rune, la chanson d'Otinel qui narre le siège de la forteresse d'Attilie, Aspremont qui tourne autour de la colline du même nom, etc.

${ }^{21}$ Voir par exemple les laisses CCCLXXI à CCCLXXIV (v. 10.060-10.310) : en 250 vers, Charlemagne passe de la Bretagne à la Saxe en traversant à plusieurs reprises la Bavière, sans oublier des retours en France et à Aix.

${ }^{22}$ Sur de curieuses anticipations romanesques, dans la littérature française des $\mathrm{XII}^{\mathrm{e}}$ et $\mathrm{XIII}^{\mathrm{e}}$ siècle, de la notion de portrait "ressemblant», voir notre article "De la représentation médiévale. Fantasme et ressemblance dans l'esthétique romanesque du Moyen Âge central», Poétique, 127 (2001), p. 259-279.
} 
L'endemain, vers Gascoingne ont leur voie tenue, et ont tant chevauchié par la montaingne herbue qu'en une grant forest s'est li ost embatue, en un pas moult divers et d'entree et d'issue. Mes grant gent de Gascoigne, qui la voie ont seüe avoient des François, s'iert adonc pourveüe des Gascoins et de gens qu'el pas fu racondue: et furent embuschié en la forest plus drue, et en si divers pas et mise et descendue qu'ainz ceste oeuvre ne fu de François conneüe devant que mescheance leur fu desus corue. Mes pour ce que li oz de France estoit cremue, laissierent passer Kallons et sa gent de value, mes en l'arriere garde s'est en travers ferue ainz que li oz de france eüst lor gent veüe, et si hardiement en venant combatue a cele arriere garde et si bien maintenue qu'a ce que li François ceste oeuvre aperceüe n'avoient de riens nee, ne dont ele ert issue. Et a ce que lor gent iert auques d'armes nue, en fu tant a ce point occise et confondue c'onques Kallons n'ot mes tel perte receüe, car du commun de l'ost de la gent mieus creüe em prouece, et en bien de valeur soustenue, i perdu bien .M. hommes, dont l'ost fu coie et mue, et li rois plus que nus quant l'en amenteüe li a ce vilain fait, dont d'angoisse tressue. Lors retorna au pas, mes voie fu perdue, car aus Gascoins ne pot faire desconvenue, ainz se fust ja la gent par mainz lieus espandue, el bois et el destrois aussi haus comme nue, de quoi la gent de France devint tout esperdue, quant la gent de Gascoingne ne fu aconsseüe. (v. 8765-8797)

À aucun moment de son récit, Girart ne semble se douter qu'il raconte la bataille de Roncevaux : il s'agit certes d'une attaque dirigée contre l'arrière-garde de l'armée de Charles à son retour d'Espagne, mais les attaquants (Gascons) et le lieu (une forêt) sont si différents de ceux de la légende que la connexion entre les deux récits est impossible. Les sources, pourtant, n'indiquaient-elles pas le nom du col fatal? Et Girart peut-il avoir été abusé par ses souvenirs épiques au point de ne pas s'étonner de ne lire nulle part dans la chronique le récit de la lutte du neveu de Charlemagne contre les Sarrasins? À l'hypothèse de l'aveuglement de Girart, on serait donc tenté d'opposer l'idée que l'auteur n'est pas dupe lui-même, mais qu'il est si choqué de la réduction du grandiose combat rolandien à un guet-apens basque qu'il accentue, dans son récit, les marques de dissemblances, afin de ménager à la fois la chronique et le mythe : il n'émonde pas la première, mais coupe tous les ponts entre elle et sa résultante légendaire. Trop en contradiction avec l'horizon d'attente du lecteur 
médiéval, le récit historique de la bataille de Roncevaux est relégué au rang de péripétie grave, mais finalement sans conséquence réelle, des guerres menées par Charlemagne; surtout, le lieu est anonyme, silence dans lequel l'événement luimême finit par s'abîmer. Certes, Girart semble un instant se souvenir du grand planctus de La Chanson de Roland dans l'évocation de la douleur de Charles :

Quant Kallemaine voit comment la chose aloit

de ses hommes que mors par mi le champ veoit, tel duel en ot au cuer a poi qu'il n'enrragoit.

Mes ne dist a ses hommes lors tot quan qu'il pensoit, fors tant qu'il dist, s'il peut, ses amis vengeroit. (v. 8798-8802)

Mais le récit historique reprend ses droits dès le vers suivant, en une considération tactique de bon sens, quoique bien prosaïque de la part d'un héros épique :

Mes bien vit et aprist que, se la yvernoit, que vivres aus chevaux et a sa gent faudroit: par quoi il ot consseil qu'en France tourneroit, fors que Gascoigne avant, s'il pooit, greveroit. (v. 8803-8806)

Malheureuse, l'escarmouche sera reléguée dans le silence; ni noms de lieu, ni patronymes de combattants morts ne survivront à ce désastre et la gloire de Charlemagne n'en sera pas plus ternie que dérangée; complice à sa manière du processus mythico-littéraire qui transfigura la honteuse défaite en glorieux sacrifice, Girart laisse sans vengeance les victimes des Gascons pour mieux exalter plus loin l'apothéose épique de la mort de Roland.

$\mathrm{Au}$ chapitre des succès de l'empereur, Girart constate une autre discordance entre les récits historique et traditionnel de la guerre de Saxe. On sait que les parts respectives de cette dernière et de la guerre d'Espagne sont dans l'histoire et dans la légende inversement proportionnelles : si la campagne espagnole ne dura en réalité que quelques mois et est sans commune mesure avec les vingt ans et plus que Charlemagne employa à soumettre les Saxons, c'est cependant la guerre de Saxe qui est le parent pauvre dans la tradition épique. Girart connaissait La Chanson des Saisnes de Jean Bodel, dont il cite le nom tout à la fin du Charlemagne (vers 22.758: «Jehans Bodiaus [...] a la langue polie ») ; c'est même le seul texte épique auquel il fait explicitement allusion. On peut, si l'on veut, voir dans cette mention un hommage à l'un des plus grands écrivains du XIII ${ }^{\mathrm{e}}$ siècle, mais on peut douter de l'éclat du nom de Jean Bodel près d'un siècle après sa mort - tout au plus Girart nous prouve-t-il qu'il n'est pas encore totalement tombé dans l'oubli - et il paraît plus vraisemblable de comprendre dans cette allusion un stratagème de Girart pour évacuer un épisode embarrassant de la geste de l'empereur. En effet, dans Les Saisnes, Jean Bodel dit explicitement que son récit se situe après la bataille de Roncevaux : Roland mort, le héros sera son frère, Baudouin. Vainqueur de Guiteclin (l'historique Witukind), dont il séduit et épouse la femme Sebile avant de mourir héroïquement de la main des fils de Guiteclin, que Charlemagne finira évidemment par vaincre, Baudouin est le nouveau Roland; on ignore si la promotion héroïque du personnage est le fait de Jean Bodel ou de sa source, mais ce qui est certain c'est que 
les conteurs médiévaux ne sont pas d'accord sur son lien de parenté exact avec l'empereur. Le personnage est issu de La Chanson de Roland où il est cité à deux reprises (vers 314 et 363) comme fils de Ganelon, ce qui ne ferait donc de lui que le demi-frère de Roland. Jean Bodel, au contraire, le présente comme frère germain du martyr de Roncevaux ${ }^{23}$. Pour Girart d'Amiens, la solution du problème ne fait aucun doute ; on lit en effet dans le Charlemagne:

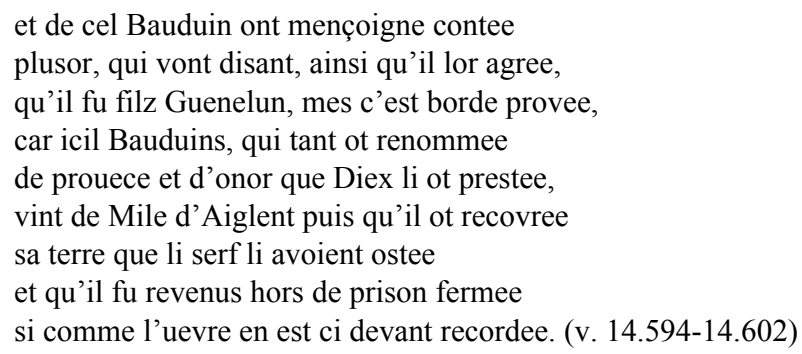

L'argumentation est claire : un héros aussi positif que Baudouin ne saurait appartenir au lignage honni de Ganelon ${ }^{24}$, à cette «geste» dont Bertrand de Bar-sur-Aube fait descendre tous les barons révoltés, dans un prologue célèbre ${ }^{25}$ qui, plutôt que trois «matières » narratives, nous détaille les caractéristiques des trois actants principaux du drame épique ${ }^{26}$. On remarque en passant que parmi les conteurs indignes de foi figure le narrateur du manuscrit d'Oxford du Roland! Preuve, s'il en était besoin, que les accusations de mensonge des conteurs médiévaux ne tiennent nul compte de l'ancienneté des traditions dont elles se prévalent et ne visent jamais qu'à asseoir

${ }^{23}$ Cf Jehan Bodel, La Chanson des Saisnes, éd. Annette Brasseur, Genève, Droz, «Textes littéraires français », 1989, v. 1262 (ms. A) : « Son neveu Baudoïn qui freres fu Rollant». Jean Bodel n'est pas plus précis, mais en l'absence totale de référence à Ganelon, on peut supposer qu'il appuie l'idée que lui prête Girart d'Amiens de faire de Baudouin le double parfait de Roland.

${ }^{24} \mathrm{Il}$ est à peine besoin de préciser que Girart ne connaît pas (ou ne veut pas connaître) les traditions qui font de Roland un fils incestueux de Charlemagne. Voir, à ce sujet, G. J. Brault, "The Legend of Charlemagne's Sin in Girart d'Amiens », Romance Notes, 4 (1962-1963), p. $72-76$.

${ }_{25}^{25}$ «'ot que trois gestes en France la garnie ; [...] Des rois de France est la plus seignorie, [...] Et l'autre aprés, bien est droit que jeu die, / Fu de Doon a la barbe florie, / Cil de Maience qui molt ot baronnie. / De ce lingnaje, ou tant ot de boidie, / Fu Ganelon qui, par sa tricherie, / En grant dolor mist France la garnie, / [...] La tierce geste, qui molt fist a prisier, / Garin de Monglenne au vis fier ». (Bertrand de Bar-sur-Aube, Girart de Vienne, éd. par Wolfgang Van Emden, Paris, Société des Anciens Textes Français, 1977, v. 11, 13-16, 21-23 et 46-47).

${ }^{26}$ Actants qui se calquent sur l'analyse narratologique élémentaire du texte matrice qu'est $\mathrm{La}$ Chanson de Roland, à travers les personnages de Roland (neveu de Charlemagne - le souverain), d'Olivier (petit-fils de Garin - le vassal fidèle) et de Ganelon (petit-fils de Doon le traître), dont la distribution montre exemplairement l'identification médiévale de la naissance et de l'essence. 
leurs prétentions dans une légitimité aussi absolue que fictive. Cette remarque de Girart suit un résumé de La Chanson des Saisnes, sans doute motivé par l'embarras de l'auteur qui, ayant consciencieusement copié le contenu des Grandes Chroniques de France, se trouve fort emprunté pour concilier le récit historique et le texte de Jean Bodel. Non seulement les épisodes de la chanson de geste n'ont rien à voir avec le récit du chroniqueur, mais Jean Bodel est formel sur le chronotope de sa propre narration: Roland est mort lorsque Charlemagne envahit la Saxe. Girart, qui, pour des raisons tant intertextuelles (son obédience à la Chronique de Turpin) que dramatiques (quel plus bel apogée imaginer à la carrière du grand empereur?), ne conçoit pas son Charlemagne se terminant autrement que par la bataille de Roncevaux, résume donc en quelques vers le contenu de La Chanson des Saisnes et résout les contradictions des deux narrations en les séparant résolument et en affirmant qu'il y avait en fait deux Guiteclins, ce qui est historiquement controuvé :

Quant Guitequins fu mors, la guerre fu cessee

un grant temps en Saissone, mes puis renovelee

refu par un sien fil, un roy de grant posnee

qui de Saissone tint adonques la contree:

Guitequins ot a nom, celui en cui trouvee

iert largesce et honor tant com il ot duree.

Et fu cil Guitequins qui puis ot espousee

Sebile la roÿne, la plus tres bele nee

que l'en peüst trouver dusqu'en la mer betee.

Ce fu cele Sebile par qui Rune passee

refu par Bauduin qui lors l'avoit amee; (v. 14.583-14.593)

Nous approchons ici de la fin du livre II ; le «grant temps» de paix en Saxe est évidemment destiné à accueillir le récit des combats en Espagne, qui empliront le livre III de leur bruit et de leur fureur.

Après le récit de la bataille de Roncevaux, et avant de conclure sa chanson par la mort de Charlemagne, il suffira donc à Girart de dire qu'en cet endroit devrait se placer la guerre de Baudouin contre Guiteclin II, mais que Jean Bodel l'a si bien racontée qu'il n'est pas nécessaire d'en refaire ce récit :

Vous avez bien oÿ comment fu convertie Sessonne par Kallon, et a lui aloïe,

et la gent du paÿs en sainz fons baptizie;

mais par Guitequin fu arriere renoïe,

le fil de Guitequin le Fel, qui en sa vie,

n'ot ainz de faire bien courage ni anvie.

Mais ses filz fu uns homs plains de chevalerie,

par quoi Sessonne mist toute en sa seignorie,

et prist bele moillier et noble et seignorie

qu'au frere Rollant fu, Baudouÿn, puis amie,

tout ainssi com on dist, quar ceste estoire mie

en cronique ne truis, ne mainte chevauchie

que Kallemaine fist; mais ainz seront fallie 


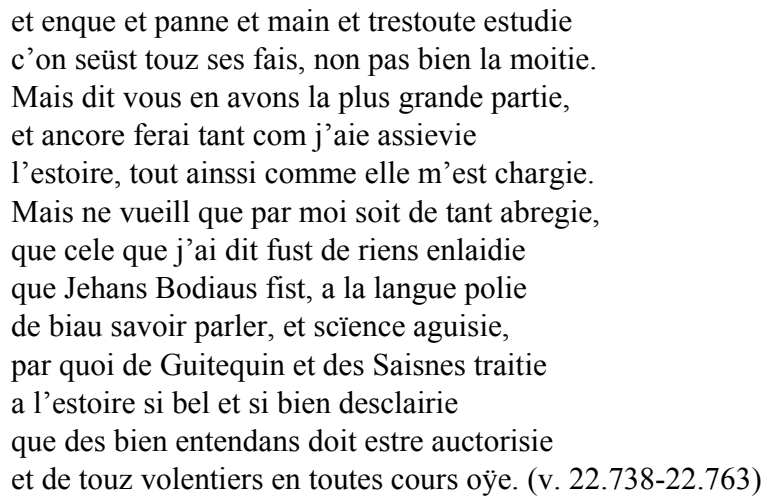

On aura aussi noté l'aveu des vers 22.748-22.749 ! Ici encore Girart concilie histoire et légende : les chroniqueurs nous racontent véridiquement la guerre de Saxe, mais ils n'ont pas tout dit. En même temps, il concilie les légendes entre elles: les chansons de geste du cycle de l'expédition espagnole situant toujours Roncevaux à l'horizon lointain et menaçant de leur propre récit (prétexte pour déplorer à l'avance la mort d'Olivier et de Roland et envoyer quelques anathèmes au félon Ganelon en des prolepses néo-scripturaires ${ }^{27}$ ), Girart ressent comme une incongruité, voire comme un blasphème, l'idée que Charlemagne, privé de son plus que fils, ait pu continuer ses campagnes avec d'autres combattants, fussent-ils en tout pareils au neveu mort. Sans réfuter le récit de Jean Bodel, Girart d'Amiens le renvoie à sa célébrité pour mieux l'évacuer.

On aura remarqué ici, comme dans la citation du livre II, le portrait flatteur que Girart fait de Guiteclin II : autant le père («le Fel») était sauvage, autant le fils est raffiné. Notre auteur semble avoir été frappé plus que de raison par les différences de ton séparant la chronique de la chanson de geste : tout se passe en effet comme s'il avait ressenti la sécheresse du récit historique sur le même plan d'objectivité que les ornements de l'élaboration littéraire. Le départ que nous faisons naturellement entre un ennemi «brut» et un combattant idéalisé (comme le sont toujours les païens des chansons de geste, car il est de toute nécessité que les Francs affrontent des adversaires à leur mesure) n'a rien de clair pour Girart qui y voit naturellement un argument en faveur de la dichotomie qu'il propose entre deux souverains saxons se succédant comme s'ils reflétaient deux âges différents du royaume saxon, le premier primitif et barbare, le second plus moderne et, donc, plus policé $^{28}$. De la même manière que la misérable embuscade basque narrée par le chroniqueur ne pouvait être assimilée au grandiose combat de Roncevaux, le mesquin Guiteclin de l'histoire ne peut rien avoir de commun avec le preux mari de la reine Sebile. Encore une fois, on pourrait se demander si Girart ne travaille pas plus pour ses lecteurs «informés», dont il prévient les interrogations et les doutes,

\footnotetext{
${ }^{27}$ Ganelon est, comme Judas, celui «ki la traïson fist» (La Chanson de Roland, éd. Cesare Segre, Genève, Droz, «Textes littéraires français », 1989, v. 178).

${ }^{28}$ Est-ce aller trop loin que de remarquer que l'idée de la rude et puissante "barbarie » primitive s'avère ici toute formée, et ce bien avant le Romantisme?
} 
que pour lui-même, et si ses acrobaties narratives n'obéissent pas à un dessein très concerté : à savoir, en accueillant toutes les traditions et en tentant d'en arranger la chronologie relative et la valeur exemplaire, de rehausser la chronique par la littérature, mouvement qui pourrait nous paraître paradoxal, mais qui nous prouve une fois de plus que la vérité médiévale n'est rien sans la reconnaissance de la tradition. Habitués que nous sommes à voir les modernes auteurs de romans historiques s'astreindre à un harassant travail de documentation afin de convaincre leurs lecteurs de la valeur de leur entreprise, nous ne pouvons qu'être fascinés par l'exemple de Girart d'Amiens, aux yeux de qui tous les raffinements de l'art littéraire ne l'emporteront jamais sur la certitude d'une vérité révélée. Écartelé entre la chronique et la légende, son Charlemagne reste le héros intemporel sur qui repose la mémoire infrangible de la civilisation occidentale.

Alain Corbellari

Université de Lausanne 